\title{
ON EXTREME X-HARMONIC FUNCTIONS
}

\author{
E. B. DYNKIN
}

\begin{abstract}
All positive harmonic functions in an arbitrary domain $E$ of a Euclidean space can be decomposed in a unique way into extreme functions. The latter can be obtained by a passage to the limit from $k^{y}(x)=\frac{g(x, y)}{g(a, y)}$ where $g(x, y)$ is the Green function of the Laplacian and $a$ is a fixed point of $E$. Our goal is to get similar results for a class of positive functions on a space of measures. These functions are associated with a superdiffusion $X$ and we call them $X$-harmonic. Denote $\mathcal{M}_{c}(E)$ the set of all finite measures $\mu$ supported by compact subsets of $E$. $X$-harmonic functions are functions on $\mathcal{M}_{c}(E)$ characterized by a mean value property formulated in terms of exit measures of a superdiffusion. Extreme $X$ harmonic functions play the same role as their classical counterpart. We describe a limit process for getting these functions. Instead of the ratio $\frac{g(x, y)}{g(a, y)}$ we use a Radon-Nikodym derivative of the probability distribution of an exit measure of $X$ with respect to the probability distribution of another such measure.
\end{abstract}

\section{Introduction}

1.1. $X$-harmonic functions. Suppose that $L$ is a second order uniformly elliptic operator in a domain $E$ of $\mathbb{R}^{d}$. An $L$-diffusion is a continuous strong Markov process $\xi=\left(\xi_{t}, \Pi_{x}\right)$ in $E$ with the generator $L$. A function $h$ in a domain $E$ is called $\xi$-harmonic (or $L$-harmonic) if, for every domain $D \Subset E,{ }^{1}$

$$
\Pi_{x} h\left(\xi_{\tau_{D}}\right)=h(x) \text { for all } x \in D \text {. }
$$

Here $\tau_{D}$ is the first exit time of $\xi$ from $D$. This condition is satisfied if and only if $L h=0$ in $E$.

Let $\psi$ be a function from $E \times \mathbb{R}_{+}$to $\mathbb{R}_{+}$where $\mathbb{R}_{+}=[0, \infty)$. An $(L, \psi)$ superdiffusion is a model of an evolution of a random cloud. It is described by a family of random measures $\left(X_{D}, P_{\mu}\right)$ where $D \subset E$ and $\mu$ is a finite measure on $E$. ${ }^{2}$ If $\mu$ is concentrated on $D$, then $X_{D}$ is concentrated on $\partial D$. We call $X_{D}$ the exit measure from $D$. Heuristically, it describes the mass distribution on an absorbing barrier placed on $\partial D$.

We put $\mu \in \mathcal{M}_{c}(D)$ if $\mu$ is a finite measure concentrated on a compact subset of $D$. We say that a function $H: \mathcal{M}_{c}(E) \rightarrow \mathbb{R}_{+}$is $X$-harmonic and we write $H \in \mathbb{H}(X)$ if, for every $D \Subset E$ and every $\mu \in \mathcal{M}_{c}(D)$,

$$
P_{\mu} H\left(X_{D}\right)=H(\mu) \text {. }
$$

Received by the editors January 9, 2005.

The author is partially supported by National Science Foundation Grant DMS-0204237.

${ }^{1}$ We write $D \Subset E$ if $D$ is a bounded domain such that the closure $\bar{D}$ of $D$ is contained in $E$.

${ }^{2}$ Assumptions about these random measures are formulated in Section 1.2. 
For every domain $D \subset E$ we have an inclusion $\mathcal{M}_{c}(D) \subset \mathcal{M}_{c}(E)$. We say that $H$ is $X$-harmonic in $D$ if

$$
P_{\mu} H\left(X_{O}\right)=H(\mu) \text { for all } O \Subset D, \mu \in \mathcal{M}_{c}(O) .
$$

An element $H$ of $\mathbb{H}(X)$ is called extreme if the conditions $\tilde{H} \leq H, \tilde{H} \in \mathbb{H}(X)$ imply that $\tilde{H}=$ const. $H$.

Fix $a \in E$ and denote by $\mathbb{H}(X, a)$ the class of all positive $X$-harmonic functions $H$ such that $H\left(\delta_{a}\right)=1\left[\delta_{a}\right.$ is the unit mass concentrated at $\left.a\right]$. Let $\mathbb{H}_{e}(X, a)$ stand for the set of all extreme elements that belong to $\mathbb{H}(X, a)$. According to Theorem 3.1 in [Dyn04a], the formula

$$
H(\mu)=\int \hat{H}(\mu) \nu(d \hat{H})
$$

establishes a 1-1 correspondence between $H \in \mathbb{H}(X, a)$ and probability measures $\nu$ on $\mathbb{H}_{e}(X, a){ }^{3}$

1.2. Superdiffusions. We write $f \in \mathcal{B}$ if $f$ is a positive $\mathcal{B}$-measurable function. We denote by $\mathcal{B}(E)$ the class of all Borel subsets of $E$ and by $\mathcal{M}(E)$ the set of all finite measures on $\mathcal{B}(E)$.

Suppose that to every open set $D \subset E$ and every $\mu \in \mathcal{M}(E)$ there corresponds a random measure $\left(X_{D}, P_{\mu}\right)$ on $\mathbb{R}^{d 4}$ such that, for every $f \in \mathcal{B}(E)$,

$$
P_{\mu} e^{-\left\langle f, X_{D}\right\rangle}=e^{-\left\langle V_{D}(f), \mu\right\rangle}
$$

where $u=V_{D}(f)$ satisfies the equation ${ }^{5}$

$$
u+G_{D} \psi(u)=K_{D} f .
$$

Here

$$
\begin{aligned}
& G_{D} f(x)=\Pi_{x} \int_{0}^{\tau_{D}} f\left(\xi_{s}\right) d s, \\
& K_{D} f(x)=\Pi_{x} 1_{\tau_{D}<\infty} f\left(\xi_{\tau_{D}}\right)
\end{aligned}
$$

are the Green operator and the Poisson operator of $\xi$ in $D$. We call the family $X=\left(X_{D}, P_{\mu}\right)$ an $(L, \psi)$-superdiffusion if, besides (1.4)-(1.5) it satisfies the following condition.

1.2.A. [Markov property] For every $\mu \in \mathcal{M}_{c}(E)$ and every $D \Subset E$,

$$
P_{\mu} Y Z=P_{\mu}\left(Y P_{X_{D}} Z\right)
$$

if $Y \geq 0$ is measurable with respect to the $\sigma$-algebra $\mathcal{F}_{\subset D}$ generated by $X_{O}, O \subset$ $D$ and $Z \geq 0$ is measurable with respect to the $\sigma$-algebra $\mathcal{F}_{\supset D}$ generated by $X_{O^{\prime}}, O^{\prime} \supset D$.

\footnotetext{
${ }^{3}$ In Section 4.1 we deduce the representation (1.3) from a result in [Dyn78].

${ }^{4} \mathrm{~A}$ random measure on a measurable space $\left(S, \mathcal{B}_{S}\right)$ is a pair $(X, P)$ where $X(\omega, B)$ is a kernel from an auxiliary measurable space $(\Omega, \mathcal{F})$ to $\left(S, \mathcal{B}_{S}\right)$ and $P$ is a probability measure on $\mathcal{F}$. [We say that $p(x, B), x \in E, B \in \mathcal{B}^{\prime}$ is a kernel from a measurable space $(E, \mathcal{B})$ to a measurable space $\left(E^{\prime}, \mathcal{B}^{\prime}\right)$ if it is a $\mathcal{B}$-measurable function in $x$ and a finite measure in $B$.]

${ }^{5} \psi(u)$ is a short writing for $\psi(x, u(x))$.
} 
The existence of a $(L, \psi)$-superdiffusion is proved for a convex class of functions $\psi$ which contains functions $\psi(x, u)=b(x) u^{\alpha}$ with bounded positive Borel $b$ and $1<\alpha \leq 2$. [See, e. g., Chapter 4 in [Dyn02].] It follows from (1.6)-(1.4) that

$$
P_{\mu}\left\{X_{D}(D)=0\right\}=1
$$

and

$$
P_{\mu}\left\{X_{D}=\mu\right\}=1 \quad \text { if } \mu(D)=0 .
$$

Let $\mathcal{F}$ stand for the $\sigma$-algebra in $\Omega$ generated by $X_{D}(B)$ where $D \Subset E$ and $B \in \mathcal{B}(E)$. Denote by $\mathfrak{M}$ the $\sigma$-algebra in $\mathcal{M}_{c}(E)$ generated by the functions $F(\mu)=\mu(B)$ with $B \in \mathcal{B}(E)$. If $\mu \in \mathcal{M}_{c}(E)$ and $D \Subset E$, then, $P_{\mu}$-a.s., $X_{D} \in \mathcal{M}_{c}(E)$ and $X_{D}$ is a measurable mapping from $(\Omega, \mathcal{F})$ to $\left(\mathcal{M}_{c}(E), \mathfrak{M}\right)$. Moreover, if $\mu \in \mathcal{M}_{c}(D)$, then, $P_{\mu}$-a.s., $X_{D} \in \mathcal{M}(\partial D)$. It follows from (1.4) that $H(\mu)=P_{\mu} Y$ is $\mathfrak{M}$-measurable for every $\mathcal{F}$-measurable $Y \geq 0$.

We have:

1.2.B. [Absolute continuity property] For every set $C \in \mathcal{F}_{\supset D}$ either $P_{\mu}(C)=$ 0 for all $\mu \in \mathcal{M}_{c}(D)$ or $P_{\mu}(C)>0$ for all $\mu \in \mathcal{M}_{c}(D)$.

A proof of this property can be found in [Dyn04b], Theorem 5.3.2.

1.3. $H$-transform. Let $X=\left(X_{D}, P_{\mu}\right)$ be a superdiffusion in $E$ and let $E$ be the union of $U_{k}$ such that $U_{1} \Subset U_{2} \Subset \ldots U_{k} \Subset \ldots$ Put $\mathcal{M}=\mathcal{M}_{c}(E)$ and denote by $\mathcal{O}_{k}$ the class of all open sets $D \Subset U_{k}$.

The space $(\mathcal{M}, \mathfrak{M})$ is a measurable Luzin space. ${ }^{6}$ Therefore Kolmogorov's extension theorem is applicable to $\mathcal{M}^{\mathcal{O}_{k}}$. Fix $a \in E$ and $H \in \mathbb{H}(X, a)$. Put $P_{a}=P_{\delta_{a}}$ and consider a family

$$
M_{n, k}\left(D_{1}, C_{1} ; \ldots ; D_{n}, C_{n}\right)=P_{a}\left\{X_{D_{1}} \in C_{1}, \ldots, X_{D_{n}} \in C_{n} ; H\left(X_{U_{k}}\right)\right\}
$$

where $n=1,2, \ldots, D_{1}, \ldots, D_{n} \in \mathcal{O}_{k}$ and $C_{1}, \ldots, C_{n} \in \mathcal{M}$. ${ }^{7}$ Note that for $n>1$

$$
M_{n, k}\left(D_{1}, C_{1} ; \ldots ; D_{n-1}, C_{n-1} ; D_{n}, \mathcal{M}\right)=M_{n-1, k}\left(D_{1}, C_{1} ; \ldots ; D_{n-1}, C_{n-1}\right)
$$

Since $H \in \mathbb{H}(X, a), M_{1, k}(D, \mathcal{M})=1$ if $a \in U_{k}$. By (1.8), this is true also if $a \notin U_{k}$. By Kolmogorov's theorem, there exists a probability measure $P_{a, k}^{H}$ on $\mathcal{M}^{\mathcal{O}_{k}}$ such that, for all $D_{1}, \ldots, D_{n} \in \mathcal{O}_{k}$ and $C_{1}, \ldots, C_{n} \in \mathcal{M}$

$$
\begin{aligned}
P_{a, k}^{H}\left\{X_{D_{1}} \in C_{1}, \ldots, X_{D_{n}} \in\right. & \left.C_{n}\right\}=M_{n, k}\left(D_{1}, C_{1} ; \ldots ; D_{n}, C_{n}\right) \\
& =P_{a}\left\{X_{D_{1}} \in C_{1}, \ldots, X_{D_{n}} \in C_{n} ; H\left(X_{U_{k}}\right)\right\} .
\end{aligned}
$$

This implies

$$
P_{a, k}^{H} Y=P_{a}\left[Y H\left(X_{U_{k}}\right)\right]
$$

\footnotetext{
${ }^{6}$ That is there exists a $1-1$ mapping from $\mathcal{M}$ onto a Borel subset $\tilde{\mathcal{M}}$ of a compact metric space such that elements of $\mathfrak{M}$ correspond to Borel subsets of $\tilde{\mathcal{M}}$.

${ }^{7}$ Writing $P\{A ; f\}$ means $\int_{A} f d P$.
} 
for all $k$ and all $Y \in \mathcal{F}_{\subset U_{k}}$. Indeed, by (1.9), formula (1.10) holds for $Y=$ $1_{C_{1}}\left(X_{D_{1}}\right) \ldots 1_{C_{n}}\left(X_{D_{n}}\right)$ where $D_{1}, \ldots, D_{n} \in \mathcal{O}_{k}$ and these functions generate $\mathcal{F}_{\subset U_{k}}$. By 1.2.A, $P_{a}\left[Y H\left(X_{U_{k}}\right)\right]=P_{a}\left[Y H\left(X_{U_{\ell}}\right)\right]$ for $k<\ell$ and $Y \in \mathcal{F}_{\subset U_{k}}$. Since $\mathcal{O}_{k} \uparrow \mathcal{O}(E)$, there exists a measure $P_{a}^{H}$ on $\mathcal{M}^{\mathcal{O}(E)}$ which coincides with $P_{a, k}^{H}$ on $\mathcal{M}^{\mathcal{O}_{k}}$. Clearly,

$$
P_{a}^{H} Y=P_{a}\left[Y H\left(X_{U}\right)\right] \quad \text { for all } U \Subset E, Y \in \mathcal{F}_{\subset U} .
$$

The measure $P_{a}^{H}$ is called the $H$-transform of $P_{a} .{ }^{8}$

1.4. Main results. We denote by $\mathcal{P}_{D}(\mu, \cdot)$ the probability distribution of $X_{D}$ under $P_{\mu}$, that is

$$
\mathcal{P}_{D}(\mu, A)=P_{\mu}\left\{X_{D} \in A\right\} \quad \text { for } A \in \mathfrak{M} \text {. }
$$

Fix a reference point $a \in E$ and put $\mathcal{P}_{D}(A)=\mathcal{P}_{D}\left(\delta_{a}, A\right)$. By 1.2.B, there exists a Radon-Nikodym derivative

$$
H_{D}^{\nu}(\mu)=\frac{\mathcal{P}_{D}(\mu, d \nu)}{\mathcal{P}_{D}(d \nu)} .
$$

For every $\mu \in \mathcal{M}_{c}(D)$, this is a function of $\nu \in \mathcal{M}(\partial D)$ defined up to $\mathcal{P}_{D^{-}}$ equivalence. We continue it to $\mathcal{M}(E) \times \mathcal{M}(E)$ by setting $H_{D}^{\nu}(\mu)=0$ off $\mathcal{M}_{c}(D) \times$ $\mathcal{M}(\partial D)$.

Theorem 1.1. There exists a version of $H_{D}^{\nu}(\mu)$ which is $\mathfrak{M} \times \mathfrak{M}$-measurable and $X$-harmonic in $\mu$ in the domain $D$ for every $\nu \in \mathcal{M}(\partial D)$.

In Theorems 1.2 and $1.3, H_{D}^{\nu}(\mu)$ is the version of the Radon-Nikodym derivative (1.11) described in Theorem 1.1.

We say that a sequence $D_{k}$ exhausts $E$ if $D_{1} \Subset D_{2} \Subset \ldots D_{k} \Subset \ldots$ and $E$ is the union of $D_{k}$.

Theorem 1.2. If $H \in \mathbb{H}_{e}(X, a)$, then, for every $\gamma \in \mathcal{M}_{c}(E)$ and for every sequence $D_{k}$ exhausting $E$,

$$
H(\gamma)=\lim _{k \rightarrow \infty} H_{D_{k}}^{X_{D_{k}}}(\gamma) \quad P_{a}^{H}-a . s .
$$

Theorem 1.3. Let $H$ and $D_{k}$ be the same as in Theorem 1.2 and let $M_{k}^{\mu}(\cdot)=$ $\mathcal{P}_{D_{k}}(\mu, \cdot)$. There exists a sequence $\nu_{n} \in \partial D_{n}$ such that, for every $\mu \in \mathcal{M}_{c}(E)$ and for every $k$,

$$
M_{k}^{\mu}\left\{\gamma: H_{D_{n}}^{\nu_{n}}(\gamma) \rightarrow H(\gamma) \quad \text { as } n \rightarrow \infty\right\}=1
$$

\footnotetext{
${ }^{8}$ J. L. Doob introduced $h$-transforms associated with excessive functions $h$ for a Markov process. This is an important tool in the probabilistic analysis.
} 
1.5. Comparison with the Martin boundary theory. In 1941 Martin studied positive harmonic functions in an arbitrary domain $E \subset \mathbb{R}^{d}$. Denote by $\mathcal{H}(a)$ the set of all such functions equal to 1 at $a \in E$. Martin proved that every $h \in \mathcal{H}(a)$ has a unique integral representation

$$
h(x)=\int \hat{h}(x) \mu(d \hat{h})
$$

where $\mu$ is a probability measure on the set $\mathcal{H}_{e}(a)$ of all exreme points of $\mathcal{H}(a)$. Formula (1.3) provides a counterpart of this result for $X$-harmonic functions.

A central role in the Martin theory is played by the function $k^{y}(x)=\frac{g(x, y)}{g(a, y)}(g$ is the Green function of the Laplacian in $E$ ). In terms of the Brownian motion $\left(\xi_{t}, \Pi_{x}\right)$ it can be expressed by the formula

$$
k^{y}(x)=\frac{g(x, d y)}{g(a, d y)}
$$

where

$$
g(x, B)=\Pi_{x} \int_{0}^{\tau_{E}} 1_{B}\left(\xi_{t}\right) d t .
$$

Note an obvious similarity between (1.14) and (1.11). If $D \Subset E$, then $k^{y}(x)$ is harmonic in $D$ for every $y \in \partial D$. Theorem 1.1 establishes a similar property of $H_{D}^{\nu}(\mu)$.

Theorem 1.2 is a counterpart of the following proposition: ${ }^{9}$

1.5.A. If $D_{n}$ exhaust $E$ and if $h \in \mathcal{H}_{e}(a)$, then

$$
h(x)=\lim k^{\xi_{\tau_{n}}}(x) \quad \Pi_{a}^{h} \text {-a.s.. }
$$

Here $\Pi_{a}^{h}$ is the $h$-transform of $\Pi_{a}$ and $\tau_{n}$ is the first exit time from $D_{n}$.

Proposition 1.5.A in combination with the Harnack's inequality implies:

1.5.B. If $D_{n}$ exhaust $E$ and if $h \in \mathcal{H}_{e}(a)$, then there exist $y_{n} \in \partial D_{n}$ such that

$$
h(x)=\lim k^{y_{n}}(x) \quad \text { for all } x \in E .
$$

We would like to prove that, if $H \in \mathbb{H}_{e}(X, a)$, then there exist $\nu_{n} \in \mathcal{M}\left(\partial D_{n}\right)$ such that

$$
H(\mu)=\lim H_{D_{n}}^{\nu_{n}}(\mu) \quad \text { for all } \mu \in \mathcal{M}_{c}(E) .
$$

Theorem 1.3 is a weaker statement. It implies only that (1.15) holds if, for some $k$, the functions $H_{n}=H_{D_{n}}^{\nu_{n}}, n>k$ were uniformly $M_{k}^{\mu}$-integrable. Indeed, since $H_{n}$ is $X$-harmonic in $D_{n}$, we have

$$
\int H_{n}(\gamma) \mathcal{M}_{k}^{\mu}(d \gamma)=P_{\mu} H_{n}\left(X_{D_{k}}\right)=H_{n}(\mu) \quad \text { for every } n>k
$$

\footnotetext{
${ }^{9}$ We refer for the proof to [Dyn02], Chapter 7.
} 
By (1.13) and (1.16),

$$
\begin{aligned}
H(\mu)=P_{\mu} H\left(X_{D_{k}}\right)=\int H(\gamma) M_{k}^{\mu}(d \gamma) & =\int \lim H_{n}(\gamma) M_{k}^{\mu}(d \gamma) \\
& =\lim \int H_{n}(\gamma) M_{k}^{\mu}(d \gamma)=\lim H_{n}(\mu)
\end{aligned}
$$

However we do not know, if the condition of the uniform integrability of $H_{n}$ is satisfied.

The set $\mathcal{H}_{e}(a)$ can be interpreted as the exit space for $\xi$. Proposition 1.5.B is applicable to a wide class of Markov processes $\xi$ and it allows to describe the exit spaces for a number of interesting processes. [See, for instance, [Dyn64] and [Dyn66].] To apply, in a similar way, Theorem 1.3, it is necessary to learn more about the functions $H_{D}^{\nu}(\mu)$.

\section{Proof of Theorem 1.1}

The $\sigma$-algebra $\mathfrak{M}$ is countably generated. ${ }^{10}$ The existence of $\mathfrak{M} \times \mathfrak{M}$ measurable version of $H_{D}^{\nu}(\mu)$ follows from Theorem A.1 in the Appendix. Let us prove that this version can be chosen to be $X$-harmonic in $\mu$ in the domain $D$.

First we prove that, if $H_{D}^{\nu}(\mu)$ is $\mathfrak{M} \times \mathfrak{M}$-measurable, then

$$
P_{\mu} H_{D}^{\nu}\left(X_{D}\right)=H_{D}^{\nu}(\mu) \text { for } \mathcal{P}_{D^{-}} \text {-almost all } \nu \text {. }
$$

Indeed, for every $A \in \mathfrak{M}$,

$$
\mathcal{P}_{D}(\mu, A)=\int_{A} \mathcal{P}_{D}(d \nu) H_{D}^{\nu}(\mu), \quad \mathcal{P}_{D}\left(X_{O}, A\right)=\int_{A} \mathcal{P}_{D}(d \nu) H_{D}^{\nu}\left(X_{O}\right) .
$$

Therefore, by 1.2.A,

$$
\begin{aligned}
& P_{\mu} \int_{A} \mathcal{P}_{D}(d \nu) H_{D}^{\nu}\left(X_{O}\right)=P_{\mu} \mathcal{P}_{D}\left(X_{O}, A\right) \\
& =P_{\mu} P_{X_{O}}\left\{X_{D} \in A\right\}=P_{\mu}\left\{X_{D} \in A\right\}=\mathcal{P}_{D}(\mu, A)=\int_{A} \mathcal{P}_{D}(d \nu) H_{D}^{\nu}(\mu) .
\end{aligned}
$$

The function $H_{D}^{\nu}\left(X_{O}(\omega)\right)$ is $\mathfrak{M} \times \mathcal{F}$-measurable. By Fubini's theorem, it follows from (2.2) that

$$
\int_{A} \mathcal{P}_{D}(d \nu) P_{\mu} H_{D}^{\nu}\left(X_{O}\right)=\int_{A} \mathcal{P}_{D}(d \nu) H_{D}^{\nu}(\mu)
$$

for all $A \in \mathfrak{M}$ which implies (2.1).

To prove Theorem 1.1, we consider any $\mathfrak{M} \times \mathfrak{M}$ measurable version $\tilde{H}_{D}^{\nu}$ of the Radon-Nikodym derivative (1.6) and we put

$$
H_{D}^{\nu}(\mu)=P_{\mu} \tilde{H}_{D}^{\nu}\left(X_{D}\right)
$$

\footnotetext{
${ }^{10}$ Every uncountable Luzin measurable space is isomorphic to the unit interval $[0,1]$ with the Borel $\sigma$-algebra. This is proved, for instance, in [DY79], Appendix 1.
} 
This function is $\mathfrak{M} \times \mathfrak{M}$-measurable. By (2.1) it coincides, for $\mathcal{P}_{D^{-}}$-almost all $\nu$ with $\tilde{H}_{D}^{\nu}(\mu)$. By the Markov property 1.2.A,

$$
P_{\mu} H_{D}^{\nu}\left(X_{O}\right)=P_{\mu} P_{X_{O}} \tilde{H}_{D}^{\nu}\left(X_{D}\right)=P_{\mu} \tilde{H}_{D}^{\nu}\left(X_{D}\right)=H_{D}^{\nu}(\mu)
$$

for every $O \Subset D, \mu \in \mathcal{M}_{c}(O)$.

To prove Theorem 1.2 we need some preparations.

\section{Exit laws for Markov chains}

3.1. Markov chains. Suppose $\left(E_{n}, \mathcal{B}_{n}\right), n=0,1,2, \ldots$ is a sequence of measurable spaces. A Markov transition function is a family of kernels $p(r, x ; n, B)$, $0 \leq r<n$ from $\left(E_{r}, \mathcal{B}_{r}\right)$ to $\left(E_{n}, \mathcal{B}_{n}\right)$ such that

$$
p\left(r, x ; n, E_{n}\right)=1 \quad \text { for all } r<n, x \in E_{r}
$$

and

$$
\int_{E_{k}} p(r, x ; k, d y) p(k, y ; n, B)=p(r, x: n, B)
$$

for all $r<k<n$ and all $x \in E_{r}, B \in \mathcal{B}_{n}$.

A sequence $\omega=\left\{x_{o}, x_{1}, \ldots x_{n}, \ldots\right\}$ where $x_{n} \in E_{n}$ is called a path. Consider the space $\Omega$ of all paths and denote by $\mathcal{F}_{\leq r}\left[\mathcal{F}_{\geq r}\right]$ the $\sigma$-algebra in $\Omega$ generated by $\left\{X_{n}(\omega) \in B_{n}\right\}$ with $B_{n} \in \mathcal{B}_{n}$ and $n \leq r[n \geq r]$. By Kolmogorov's theorem, to every $x \in E_{0}$ there corresponds a probability measure $\mathbb{P}_{x}$ on $\mathcal{F}_{\geq 0}$ such that

$$
\mathbb{P}_{x}\left\{X_{0}=x\right\}=1
$$

and

$$
\begin{aligned}
\mathbb{P}_{x}\left\{X_{0}=r, X_{1} \in B_{1}, \ldots, X_{n}\right. & \left.\in B_{n}\right\} \\
& =\int_{B_{1}} \cdots \int_{B_{n}} p\left(0, x ; 1, d y_{1}\right) \ldots p\left(n-1, y_{n-1} ; n, d y_{n}\right)
\end{aligned}
$$

for all $n>0$ and all $B_{1} \in \mathcal{B}_{1}, \ldots, B_{n} \in \mathcal{B}_{n}$. The family $\left(X_{n}, \mathbb{P}_{x}\right)$ is a Markov chain with the transition function $p$.

3.2. Exit laws. A sequence of positive measurable functions $F^{n}(x), x \in E_{n}$ is called a $p$-exit law if

$$
\int_{E_{n}} p(m, x ; n, d y) F^{n}(y)=F^{m}(x) \quad \text { for all } m<n, x \in E_{r} .
$$

We denote $\mathcal{E}(p)$ the set of all $p$-exit laws and we put $F \in \mathcal{E}(p, a)$ if $F \in \mathcal{E}(p)$ and $F^{0}(a)=1$.

Suppose that $p$ satisfies the condition:

3.2.A. If $p(0, a ; n, B)=0$, then $p(m, x ; n, B)=0$ for all $x$ and all $m<n .^{11}$ 
Let $\mathcal{E}_{e}(p, a)$ stand for the set of all extreme elements of $\mathcal{E}(p, a)$. The formula

$$
F^{n}(x)=\int \hat{F}^{n}(x) \mu(d \hat{F})
$$

establishes a 1-1 correspondence between $F \in \mathcal{E}(p, a)$ and probability measures $\mu$ on $\mathcal{F}_{e}(p, a)$. This was proved in [Dyn78], Section 10.2.

Put

$$
\nu_{n}(A)=p(0, a ; n, A) .
$$

The Radon-Nikodym derivative

$$
\rho(m, x ; n, y)=\frac{p(m, x ; n, d y)}{\nu_{n}(d y)}
$$

can be chosen to satisfy equation

$$
\int_{E_{k}} \rho(r, x ; k, y) \nu_{k}(d y) \rho(k, y ; n, z)=\rho(r, x ; n, z)
$$

for all $r<k<n$ and all $x \in E_{r}, z \in E_{n}$.

3.3. $F$-transform. Suppose that $F \in \mathcal{E}(p, c)$. By Kolmogorov's theorem, there exists a probability measure $\mathbb{P}_{x}^{F}$ on the path space $\Omega$ such that

$$
\begin{aligned}
\mathbb{P}_{x}^{F}\left\{X_{0}=x, X_{1} \in B_{1}, \ldots,\right. & \left.X_{n} \in B_{n}\right\} \\
& =P_{x}\left\{X_{0}=x, X_{1} \in B_{1}, \ldots, X_{n} \in B_{n} ; F^{n}\left(X_{n}\right)\right\}
\end{aligned}
$$

for all $n>0$ and all $B_{1} \in \mathcal{B}_{1}, \ldots, B_{n} \in \mathcal{B}_{n}$.

The measure $\mathbb{P}_{x}^{F}$ is called the $F$-transform of $\mathbb{P}_{x}$. We have

$$
\mathbb{P}_{x}^{F} Y=P_{x} Y F^{n}\left(X_{n}\right)
$$

for every $Y \in \mathcal{F}_{\geq n}$. It is proved in [Dyn78], Section 10 that, if $F$ is an extreme element of $\mathcal{E}(p, a)$ and if $F^{r}(x)<\infty$, then

$$
F^{r}(x)=\lim _{n \rightarrow \infty} \rho\left(r, x ; n, X_{n}\right) \quad \mathbb{P}_{a}^{F} \text {-a.s. }
$$

\section{Proof of Theorems 1.2 and $\mathbf{1 . 3}$}

4.1. Markov chains associated with superdiffusions. To construct such chains we fix a sequence $D_{0}, D_{1}, \ldots$ exhausting $E$ and we put

$$
\begin{gathered}
\mathcal{M}_{0}=\mathcal{M}_{c}\left(D_{0}\right), X_{0}=\mu \in \mathcal{M}_{0}, \\
\mathcal{M}_{n}=\mathcal{M}\left(\partial D_{n}\right), X_{n}=X_{D_{n}} \quad \text { for } n \geq 1 .
\end{gathered}
$$

The Markov property 1.2.A of a superdiffusion implies that $\left(X_{n}, P_{\mu}\right)$ is a Markov chain with the transition function

$$
\mathcal{P}(r, \mu ; n, A)=P_{\mu}\left(X_{n} \in A\right), \quad 0 \leq r \leq n, \mu \in \mathcal{M}_{r}, A \subset \mathcal{M}_{n} .
$$

We call it the chain associated with the superdiffusion $\left(X_{D}, P_{\mu}\right)$.

\footnotetext{
${ }^{11}$ This property can be thought of as a probabilistic statement of the strong minimum principle.
} 
If $H$ is $X$-harmonic and if $F^{n}$ is the restriction of $H$ to $\mathcal{M}_{n}$, then $F$ is a $\mathcal{P}$-exit law. If $H \in \mathbb{H}(X, a)$ and if $a \in D_{0}$, then $F \in \mathcal{E}\left(\mathcal{P}, \delta_{a}\right)$. This way we define a mapping $j: \mathbb{H}(X, a) \rightarrow \mathcal{E}\left(\mathcal{P}, \delta_{a}\right)$. On the other hand, if $r<n$ and if $\mu \in$ $\mathcal{M}_{c}\left(D_{n}\right)$, then, by the Markov property 1.2.A, $P_{\mu} F^{n}\left(X_{n}\right)=P_{\mu} P_{X_{r}} F^{n}\left(X_{n}\right)=$ $P_{\mu} F^{r}\left(X_{r}\right)$ and therefore $P_{\mu} F^{n}\left(X_{n}\right)$ does not depend on $n \geq r$. We define $H=i(F)$ by the formula

$$
H(\mu)=P_{\mu} F^{n}\left(X_{n}\right) \quad \text { for } \mu \in \mathcal{M}_{c}\left(D_{n}\right) .
$$

Every $D \Subset E$ is contained in $D_{n}$ for sufficiently large $n$, and, by 1.2.A,

$$
P_{\mu} H\left(X_{D}\right)=P_{\mu} P_{X_{D}} F^{n}\left(X_{n}\right)=P_{\mu} F^{n}\left(X_{n}\right)=H(\mu) .
$$

Hence, $H \in \mathbb{H}(X, a)$ and we have a map $i: \mathcal{E}\left(\mathcal{P}, \delta_{a}\right) \rightarrow \mathbb{H}(X, a)$. Clearly, $i$ is the inverse for $j$ and both mappings preserve the convex structure. It follows from the Absolute continuity property 1.2.B that $\mathcal{P}$ satisfies the condition 3.2.A and therefore the integral representation (3.1) of exit laws implies the integral representation (1.3) of $X$-harmonic functions.

4.2. Proof of Theorem 1.2. If $\mu \in \mathcal{M}_{r}, A \subset \mathcal{M}_{n}$, then

$$
\mathcal{P}(r, \mu ; n, A)=\mathcal{P}_{D_{n}}(\mu, A), \quad \mathcal{P}\left(0, \delta_{a} ; n, A\right)=\mathcal{P}_{D_{n}}\left(\delta_{a}, A\right)
$$

and therefore

$$
\frac{\mathcal{P}(r, \mu ; n, d \nu)}{\mathcal{P}\left(0, \delta_{a} ; n, d \nu\right)}=H_{D_{n}}^{\nu}(\mu)
$$

On the other hand, by comparing (1.9) and (3.2), we get

$$
P_{a}^{H}=\mathbb{P}_{a}^{F} .
$$

If $\mu \in \mathcal{M}_{c}(E)$, then $\mu \in \mathcal{M}_{c}\left(D_{0}\right)$ for some $D_{0} \subset E$. Consider a sequence $D_{0}, D_{1}, \ldots$ exhausting $E$. By applying (4.2) and (4.3), we get (1.12) from (3.3).

4.3. Proof of Theorem 1.3. Every function $H_{D_{n}}^{X_{D_{n}}(\omega)}(\gamma)$ is $\mathfrak{M} \times \mathcal{F}$-measurable in $(\gamma, \omega)$ and therefore the set

$$
\mathcal{W}=\left\{(\gamma, \omega): H_{D_{n}}^{X_{D_{n}}(\omega)}(\gamma) \rightarrow H(\gamma) \quad \text { as } n \rightarrow \infty\right\}
$$

belongs to $\mathfrak{M} \times \mathcal{F}$. Put

$$
\Omega_{\gamma}=\{\omega:(\gamma, \omega) \in \mathcal{W}\}, \quad \mathcal{M}^{\omega}=\{\gamma:(\gamma, \omega) \in \mathcal{W}\}
$$

and $P=P_{a}^{H}$. By Theorem 1.2, $P\left(\Omega_{\gamma}\right)=1$ and, by Fubini's theorem,

$$
\int_{\Omega} M_{k}^{\mu}\left(\mathcal{M}^{\omega}\right) P(d \omega)=\int_{\mathfrak{M}} P\left(\Omega_{\gamma}\right) M_{k}^{\mu}(d \gamma)=1
$$

Hence the measure $P$ is concentrated on each of sets $\left\{M_{k}^{\mu}\left(\mathcal{M}^{\omega}\right)=1\right\}$ and therefore it is concentrated on their intersection. Since $P(\Omega)=1$, this intersection is not empty. 


\section{Appendix}

We need the following result

Theorem A.1. Suppose that $Q(x, d y)$ is a kernel from a measurable space $\left(X, \mathcal{A}_{X}\right)$ to a Luzin measurable space $\left(Y, \mathcal{A}_{Y}\right)$ and that $\mathcal{A}_{Y}$ is countable generated. Let $P$ be a finite measure on $\left(Y, \mathcal{A}_{Y}\right)$. If $Q(x, \cdot) \prec P(\cdot){ }^{12}$ for all $x$, then there exists a $\mathcal{A}_{X} \times \mathcal{A}_{Y}$-measurable version of the Radon-Nikodym derivative $\frac{Q(x, d y)}{P(d y)}$.

Proof. $1^{\circ}$. First, we note that, if a $\sigma$-algebra $\mathcal{A}$ is generated by the union of $\sigma$ algebras $\mathcal{A}_{1} \subset \mathcal{A}_{2} \cdots \subset \mathcal{A}_{n} \ldots$ if $\rho(y)=\frac{Q(d y)}{P(d y)}$, then the conditional mathematical expectation $\rho_{n}=P\left\{\rho \mid \mathcal{A}_{n}\right\}$ is equal to $\frac{Q_{n}(d x)}{P_{n}(d x)}$ where $P_{n}$ and $Q_{n}$ are the restrictions of $P$ and $Q$ to $\mathcal{A}_{n}$. Indeed, for every $A \in \mathcal{A}_{n}, \int_{A} \rho d P=Q(A)=\int_{A} \rho_{n} d P$. Therefore $\rho_{n} \rightarrow \rho$ off a set $C \in \mathcal{A}$ such that $P(C)=0$. A version of $\frac{Q(d x)}{P(d x)}$ can be defined as $\lim \rho_{n}$ off $C$ and a constant on $C$.

$2^{\circ}$. If a $\sigma$-algebra $\mathcal{A}$ in $Y$ is countably generated, then it is generated by a sequence of finite partitions of $Y$ into disjoint sets. Moreover, we can choose this partitions to generate a monotone increasing sequence of $\sigma$-algebras $\mathcal{A}_{n}$.

$3^{\circ}$. If $\mathcal{A}$ is generated by a partition $Y=Y_{1} \cup \cdots \cup Y_{n}$ and if $Q \prec P$, then

$$
\frac{Q(d y)}{P(d y)}=\frac{Q\left(Y_{k}\right)}{P\left(Y_{k}\right)} \quad \text { on } Y_{k}
$$

$4^{\circ}$. It is suffiient to prove our theorem for the case $Q(x, Y)=1$ for all $x$. We apply $1^{\circ}$ and $2^{\circ}$ to the $\sigma$-algebra $\mathcal{A}_{Y}$. By $3^{\circ}, \rho_{n}$ are $\mathcal{A}_{X} \times \mathcal{A}_{Y}$-measurable and an $\mathcal{A}_{X} \times \mathcal{A}_{Y}$-measurable version of $\frac{Q(x, d y)}{P(d y)}$ can be defined as in $1^{\circ}$.

\section{Acknowledgements}

The proof of Theorem A.1 was suggested by D.W.Stroock. It is more natural and much simpler than my original proof based on theorems of Lebesgue-Vitaly by De Possel. I am indebted to Dan Stroock for this suggestion and for other remarks that contributed to an improvement of the presentation.

\section{References}

[Dyn64] E. B. Dynkin, Martin boundary and non-negative solutions of a boundary value problem with oblique derivative, Uspekhi Mat. Nauk 5(119) (1964), 3-50, English translation in Russian Mathematical Surveys, 19:5(1964),1-48.

[Dyn66] , Brownian motion in certain symmetric spaces and non-negative eigenfunctions of the Laplace-Beltrami operator, Izv. Acad. Nauk SSSR 30:2 (1966), 455-478, English translation in Amer. Math. Soc. Transl., Series 2, 72(1968), 203-228.

[Dyn78] _ Sufficient statistics and extreme points, Ann. Probab. 6 (1978), 705-730.

[Dyn02] — Diffusions, Superdiffusions and Partial Differential Equations, University Lecture Series, 34. Amer. Math. Soc., Providence, R.I., 2002.

[Dyn04a] _ Harmonic functions and exit boundary of superdiffusion, J. Funct. Anal. 206 (2004), 33-68.

\footnotetext{
${ }^{12}$ We write $Q \prec P$ if $P(A)=0$ implies $Q(A)=0$.
} 
[Dyn04b] _ Superdiffusions and Positive Solutions of Nonlinear Partial Differential Equations (2004).

[DY79] E. B. Dynkin and A. A. Yushkevich, Controlled Markov Processes, Springer, BelinHeidelberg-New York, 1979.

Department of Mathematics, Cornell University, Ithaca, NY 14853

E-mail address: ebd1@cornell.edu 